\title{
Effect of genotype on chromosome variation in tissue culture of inbred and outbred rye
}

\author{
M. PUOLIMATKA $\dagger \& A$. KARP* \\ Department of Agricultural Sciences, University of Bristol, AFRC Institute of Arable Crops Research, Long Ashton \\ Research Station, Bristol BS18 9AF, U.K.
}

\begin{abstract}
To examine the effect of genotype on the nature of the cytological variation induced in culture, plants were regenerated from cultured immature embryos of six genotypes (lines A-F) of rye (Secale cereale L.). The variation observed differed among the six lines and was generally consistent with the donors. Regenerants of line A were consistent with the donor, cv. Ailés, in that chromosome translocations appeared at high frequencies. No structural or numerical variation was observed in the B chromosomes of line B. Two of the four inbred lines studied were very stable in culture. Inbred line D was also stable except for a translocation in the regenerants. In inbred line $\mathrm{F}$, variation was observed in the donors and the regenerants. An examination of chiasma frequency and distribution indicated that the regenerated plants generally had higher chiasma frequencies and more distally localized chiasmata than the donors. Line $\mathrm{F}$ was exceptional in that the regenerants had more interstitial chiasmata. The elevation in chiasma frequency was not as great, or statistically as significant, as the change in distribution. These results provide further evidence that the nature of cytological variation observed in regenerated plants is genotype-dependent and that, in addition to numerical and structural chromosome variation, shifts in chiasma frequency and distribution, and therefore in genetic recombination, can occur as a result of somaclonal variation.
\end{abstract}

Keywords: chiasma, interchange, rye, somaclonal variation.

\section{Introduction}

The occurrence of numerical and structural chromosome variation in cell cultures, and in the plants regenerated from them, is now a widely accepted component of the more general phenomenon of variation arising from in vitro culture, or somaclonal variation (Larkin \& Scowcroft, 1981). Polyploidy, aneuploidy, interchanges, deletions, inversions and chromosome fragmentation have all been described in plants regenerated from tissue cultures. The frequencies at which these changes occur vary not only in response to aspects of the tissue culture conditions, in particular the range and concentrations of growth regulators, but also from species to species, and even among cultivars of the same species (Karp, 1992). This indicates that while somaclonal variation is affected by external factors, there is also a genotypic influence, with some genotypes giving rise to more instability than others in the same culture conditions.

*Correspondence.

$\dagger$ Present address: Agricultural Research Centre, Institute of Plant Breeding, SF-31600, Jokioinen, Finland.
Among the Gramineae, rye (Secale cereale) is one of the diploid species in which chromosome variation has been frequently reported from tissue cultures (Bebeli et al., 1990). This contrasts with other diploid Gramineae, such as barley (Hordeum vulgare) (Karp et al., 1987) and Pennisetum americanum (Swedlund \& Vasil, 1985), where cytological variation appears to be minimal. One possible explanation for these discrepancies is differences in the genetic compositions of these species, particularly with respect to repetitive DNA sequences, such as those located in heterochromatin. It has been postulated that the late replicating nature of heterochromatin perturbs the cell cycle, resulting in enhanced chromosome breakage when cells are induced into division (Lee \& Phillips, 1988). Correlations between the location of tissue culture-induced breaks and the presence of heterochromatin have been demonstrated in several graminaceous species (Johnson et al., 1987; Benzion \& Phillips, 1988; Eizenga, 1989). Nevertheless, some species with little heterochromatin still show instability in culture, suggesting that other aspects of genetic composition are important (Karp, 1992). 
Rye is normally outbreeding and consequently highly heterozygous whereas many cultivated cereals are inbred and highly homozygous. When outbreeders are forcibly selfed, deleterious alleles are revealed in the progeny and selected against through their disturbance of fertility. This includes some genes whose actions upset mitotic and meiotic stability. Lines of rye which have been derived by successive inbreeding over several generations would therefore be expected to have lost some major determinants of instability, which, of course, may also be true for highly inbred cultivars.

To examine the effect of this aspect of the genetic component on somaclonal variation, the stability of six genotypes of rye in plants regenerated from immature embryos has been examined. The six genotypes include four lines which have been subjected to intensive inbreeding for over 60 years. One of these four lines was reported to show some instability at meiosis, whereas the other three were found to be stable (Rees, 1955). The other two genotypes are outbreeders whose meiotic system could already be said to be 'irregular': one being a cultivar which has high frequencies of spontaneous interchanges and the other containing $\mathrm{B}$ chromosomes. There have been previous studies describing somaclonal variation in 'standard' outbred rye (e.g. Linacero \& Vazquez, 1992). The aim of our study was not to make any direct comparisons between the inbred and outbred lines, but to investigate how the genetic make-up of the donor material influences the outcome in terms of somaclonal variation released. To make a thorough assessment of the effects of tissue culture, in addition to screening the regenerated plants for numerical and structural chromosome variation, a detailed study has been made of the frequency and distribution of genetic exchanges observed as chiasmata at meiosis. This study has revealed chromosome variation which results from passage through tissue culture in both the inbred and outbred genotypes of rye.

\section{Materials and methods}

\section{Growth of donor plant material}

Line $\mathrm{A}$ is the outbred cv. Ailés which contains reciprocal translocations (interchanges) (Candela et al., 1979, 1982; Figueiras et al., 1983). Line B is an outbred population which possesses $\mathrm{B}$ chromosomes and, together with line A, was supplied by Dr M. J. Puertas of the Department of Genetics, University Complutense, Madrid. Lines C, D, E and F were supplied by Dr R. N. Jones of the Department of Agricultural Sciences, U.C.W. Aberystwyth. They originated from a
Swedish variety of rye, Secale cereale L., Stålråg, and have been inbred since 1926 (Rees, 1955). About 50-60 seeds from each rye line were randomly selected and germinated. The plants were vernalized in a chamber $\left(7^{\circ} \mathrm{C}\right.$ day $/ 5^{\circ} \mathrm{C}$ night, r.h. 80 per cent $/ 90$ per cent, $8 \mathrm{~h}$ day-length) for 6 weeks and then grown in a greenhouse under controlled conditions. Inflorescences were bagged before anthesis. To ensure adequate seed set in the outbred line A, a few marked inflorescences were not bagged. Line B donors were not allowed to open pollinate to avoid confusion over the inheritance of the $\mathrm{B}$ chromosome.

\section{Tissue culture and plant regeneration}

Embryos were dissected irom green seeds of two to three heads per donor plant within 4 weeks after pollination. After ripening the remaining heads were harvested. Tissue culture and plant regeneration were performed as described by Bebeli et al. (1988). The identity of each regenerated shoot with respect to donor plant and embryio was clearly marked, e.g. regenerant $\mathrm{A} 1 / 2 / 3$ is shoot 3 derived from embryo 2 of donor A1. When adequate shoot and root systems had developed, the regenerants were transferred to small pots in vermiculite and grown for 3 weeks in the culture room $\left(8 \mathrm{~h}\right.$ day, temperature $18^{\circ} \mathrm{C}$ day $/ 15^{\circ} \mathrm{C}$ night, r.h. 80 per cent $/ 80$ per cent) and then vernalized as described earlier.

\section{Analysis of meiosis}

Inflorescences from each donor plant and the corresponding regenerants were collected just before emergence from the leaf sheath. They were fixed in Carnoy's fluid (absolute ethanol, chloroform and glacial acetic acid: 6:3:1), mordanted with a few drops of ferric chloride solution and stored at room temperature in the dark. Squash preparations from the anthers were made in aceto carmine. Photographs were taken using a Zeiss photomicroscope. Metaphase I (MI) and anaphase I (AI) were examined for any evidence of structural and numerical changes in chromosomes. Mean pollen mother cell (p.m.c.) chiasma frequencies and chiasma distribution were determined for a random sample of $20 \mathrm{MI}$ p.m.c.s as described in Karp \& Jones (1983).

\section{Results}

\section{Tissue culture and morphogenetic response}

Plant regeneration was achieved in all six lines but frequencies of shoot formation were low and four 
Fig. 1 Chromosome variation in the cv. Ailés line and regenerated plants. (a) Late diplotene and (b) metaphase I (MI) in donor, A7, showing heterozygosity for a translocation involving a nucleolar organizer chromosome. (c) Regenerated plant of donor A7 at MI, showing the same translocation. (d) Regenerant A22/1/1 with a translocation forming a 'frying-pan' configuration at MI. (e) Sister regenerant A22/2/1 with an alternate configuration at MI. (f) Translocation heterozygote $\mathrm{A} 22 / 5 / 1$ showing an adjacent configuration at MI. (g) Regenerant $\mathrm{A} 23 / 1 / 3$ at MI with a translocation forming an open chain. (h) Hexaploid pollen mother cell in regenerant $A 7 / 3$ / 8. Bar represents $10 \mu \mathrm{m}$.
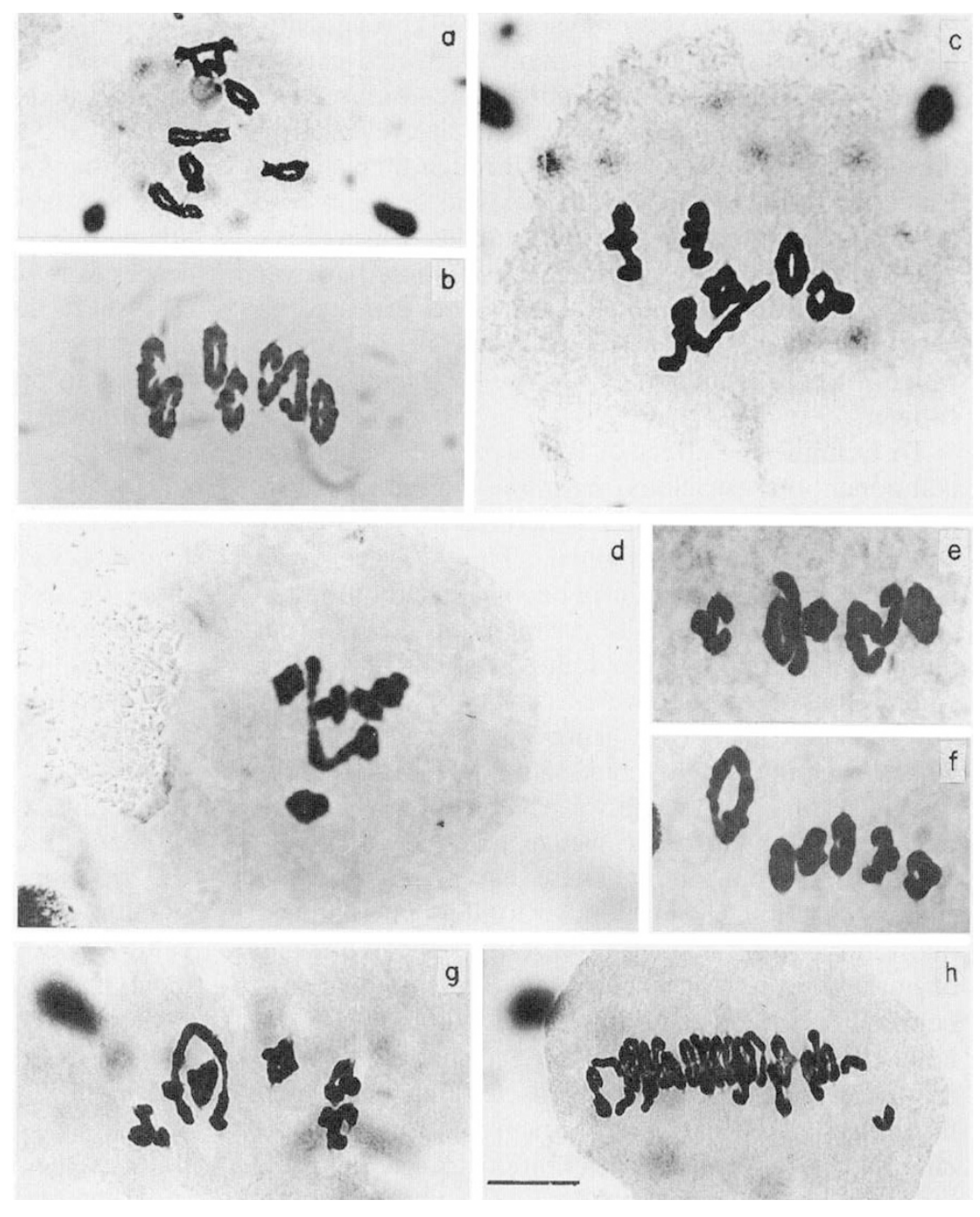

separate batches of donor plants were required to give sufficient regenerants for analysis of somaclonal variation. Over 1000 embryos were plated for each of lines $\mathrm{C}, \mathrm{D}$ and $\mathrm{E}$ and over 500 for the remaining lines. Average frequencies of shoot formation for the four batches were: 20 per cent $(A), 12$ per cent $(B), 9$ per cent $(C, D$, $\mathrm{F})$ and 7 per cent (E).

\section{Numerical and structural chromosome variation}

Line A. A total of 37 donor control plants of line A (cv. Ailés) was analysed at meiosis, of which five had been allowed to open-pollinate. Two of the 32 selfed donors (A6 and A7) were heterozygous for a reciprocal translocation (interchange). In $\mathrm{A} 7$, but not in A6, the interchange involved a nucleolus-organizer chromosome (Fig. 1a) and formed multivalents in all 20 p.m.c.s (Fig. 1b). From donor A7, 13 regenerated plants were obtained from five embryos. Three of the five embryos gave heterozygous regenerants (1/callus) with multivalent configurations consistent with that of A7 (Fig. 1c). Of the two remaining cultured embryos, one $(A 7 / 3)$ gave five plants which were only bivalent forming, although regenerant $A 7 / 3 / 8$ had a low frequency of polyploid (Fig. 1h) and aneuploid p.m.c.s. Of four regenerants from the fifth embryo $(A 7 / 6)$, three formed only bivalents and one (A7/6/7) was an interchange heterozygote. No regeneration was achieved from immature embryos of donor A6.

Four bivalent-forming donors (A2, A22, A23, A25) gave regenerants that were translocation heterozygotes. Three of these were open-pollinated (A2, A22, A23). In donor A2, one plant out of two regenerated from different embryos was heterozygous for a translocation. In donor A22, regeneration was achieved from five embryos, three of which (A22/1, A22/2, A22/5) gave regenerants which were translocation heterozygotes. The multivalent configurations 
differed in the regenerants from the different embryos, in one case (A22/1/1) forming only open chains and 'frying-pan' configurations suggestive of a non-reciprocal or very asymmetric translocation (Fig. 1d), in another (A22/2/1) forming mostly alternate configurations (Fig. 1e) and in the third $(\mathrm{A} 22 / 5 / 1)$ forming mostly open chains or adjacent rings (Fig. 1f). From donor A23, regeneration was achieved from two embryos, one of which gave three normal regenerated plants while the other gave a single regenerated translocation heterozygote (A23/1/3) (Fig. 1g). Donor A25 was selfed and gave two regenerated plants from one immature embryo. One of these was heterozygous for an asymmetric translocation which formed mostly open chains in adjacent or alternate configurations.

Line $B$. The $\mathrm{B}$ chromosome constitution of the 35 donors of line B was: 0B (10 plants), 2B (Fig. 2a) (14 plants), 4B (Fig. 2b) (10 plants) and 6B (1 plant). Regeneration was achieved from immature embryos of $0 \mathrm{~B}$ and $2 \mathrm{~B}$ plants only (selfed $4 \mathrm{~B}$ plants were sterile). One 2B donor (B22) was also heterozygous for a translocation from which four regenerants were obtained from two embryos. None of the regenerants carried the translocation. The first embryo gave two regenerated plants both with 4B chromosomes (Fig. 2c). The second embryo gave rise to one $0 \mathrm{~B}$ regenerant. When the $2 \mathrm{~B}$ donor (B22) was selfed, two progeny had $2 \mathrm{Bs}$, one carrying the translocation and the other not, two of the progeny had 4Bs and the translocation and another progeny had only the translocation. The latter was used as a donor and four plants were regenerated from one embryo. All were $0 \mathrm{~B}$ and translocation heterozygotes (Fig. 2d). No structural variation in the B chromosomes was detected in the regenerants studied.
Line C. No numerical or structural chromosome variation was observed in the 29 donor plants of inbred line C. Only 15 regenerated plants were obtained and all had regular meioses.

Line D. The 43 donor plants of line D all had normal meioses. Twelve of these donors gave normal regenerated plants. In donor D18, one regenerated plant out of three from the same embryo was a translocation heterozygote (Fig. 2e).

Line E. No structural or numerical chromosome variation was observed in the 30 donor plants of line $\mathrm{E}$ or the 16 regenerated plants obtained from nine of the donors.

Line $F$. In line $F$ all 19 donor plants analysed had normal meioses. Donor F23 gave two different variant regenerants from the same immature embryo. One of these carried a reciprocal translocation while the other had two small additional chromosomes which, as they failed to pair with the A chromosomes, were identified as putative $\mathrm{B}$ chromosomes. When the selfed progeny of donor F23 were analysed, one was identified as a translocation heterozygote but no B chromosomes were observed. A single regenerant from an immature embryo of the selfed F23 translocation heterozygote also possessed the translocation. Putative B chromosomes were also observed in an independently derived regenerant (F29/1/1) of donor F29 (Fig. 2f), although a second regenerant from the same embryo was normal. 'B' chromosomes were not observed in selfed progeny of $\mathrm{F} 29$, or in two regenerants from another immature embryo.

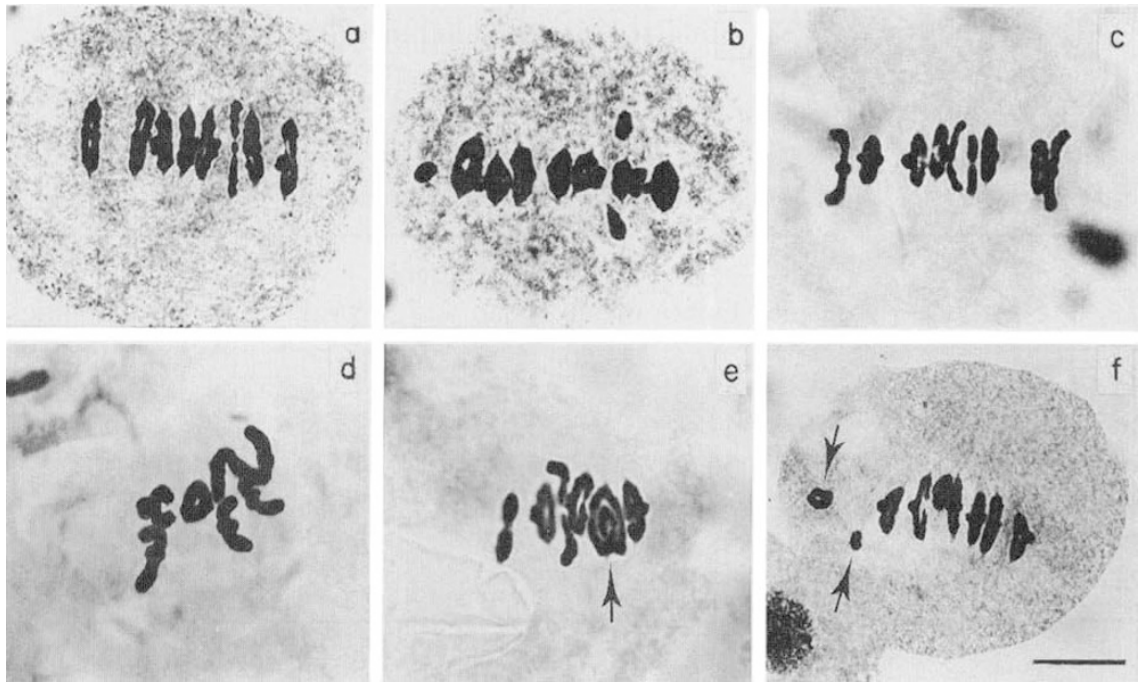

Fig. 2 Chromosome variation in donors and regenerated plants of lines $B, D$ and $F$. $(a-c)$ Donor plants of line B at $\mathrm{MI}$ of constitution $0 \mathrm{~B}(\mathrm{a}), 2 \mathrm{~B}$ (b) and 4B (c). (d) $\mathrm{MI}$ of a $0 \mathrm{~B}$ translocation heterozygote regenerated from a selfed progeny of donor B22. (e) Cultureinduced translocation in a regenerant of line D showing distinctive multivalent configuration (arrow). (f) MI of regenerant $\mathrm{F} 29 / 1 / 1$ showing $B$ chromosomes (arrows). Bar represents $10 \mu \mathrm{m}$. 


\section{Chiasma frequency and distribution}

Mean p.m.c. chiasma frequency. Mean p.m.c. chiasma frequencies were determined for each donor plant and for all the regenerated plants. In line A, to account for possible effects of inbreeding depression, chiasma frequencies were also determined in selfed progenies of donor plants. No obvious differences were observed between the selfed and outcrossed progenies in mean p.m.c. chiasma frequencies or chiasma distribution.

In line $\mathrm{A}$, mean p.m.c. chiasma frequencies ranged from 11.4 to 14.2 in the 37 donors and from 12.4 to 15.0 in the 91 regenerants. No plants were observed with exceptionally low or high values. In line $B$, there were small differences in mean p.m.c. chiasma frequencies between $0 \mathrm{~B}$ (average 12.6), $2 \mathrm{~B}$ (average 12.5) and $4 \mathrm{~B}$ (average 13.1) plants. The 19 plants regenerated from $0 \mathrm{~B}$ and $2 \mathrm{~B}$ donors ranged in mean p.m.c. chiasma frequencies from 10.7 to 13.9 . The inbred lines were generally similar and had lower mean p.m.c. chiasma frequencies compared with the outbred lines. Values ranged from 10.0 to 12.1 (line C, 29 plants), 10.2 to 13.8 (line D, 43 plants), 10.6 to 13.1 (line E, 30 plants) and 10.8 to 13.9 (line F, 19 plants). Values for regenerated plants of lines C (15 plants), D (21 plants) and $\mathrm{E}$ (16 plants) were also similar $(10.8-12.7,11.1-12.7$, 10.8-12.6, respectively). In line $F$, one plant of the nine regenerated had an exceptionally low chiasma frequency of 9.9 compared with the remainder whose values ranged between 12.2 and 13.9.

The variances in mean p.m.c. chiasma frequency among plants within the lines for the donor and regenerated plants were calculated and tested for lack of equality using a two-sided $F$ test. As shown in Table 1, there is little evidence to suggest large differences in variation between the plants of the donors and those of the regenerants. The mean chiasma frequencies were compared by pooling the variances and using a $t$-test (Table 2). In all the lines except line B, where

Table 1 Comparisons of variances for mean pollen mother cell chiasma frequencies

\begin{tabular}{|c|c|c|c|c|c|c|}
\hline Line & $\begin{array}{l}\text { Donor } \\
\text { variance }\end{array}$ & d.f. & $\begin{array}{l}\text { Regenerant } \\
\text { variance }\end{array}$ & d.f. & $F$ & Probabilities \\
\hline A & 0.3625 & 32 & 0.2704 & 90 & 1.34 & 0.284 \\
\hline B & 0.7846 & 34 & 0.9112 & 18 & 1.16 & 0.687 \\
\hline $\mathrm{C}$ & 0.2658 & 23 & 0.2926 & 13 & 1.10 & 0.810 \\
\hline $\mathrm{D}$ & 0.6115 & 38 & 0.2665 & 20 & 2.29 & 0.050 \\
\hline $\mathrm{E}$ & 0.2660 & 25 & 0.2423 & 9 & 1.10 & 0.937 \\
\hline $\mathbf{F}$ & 0.5821 & 22 & 1.5803 & 8 & 2.71 & 0.061 \\
\hline
\end{tabular}

d.f.: degrees of freedom. the means were almost identical, the regenerated plants had mean chiasma frequencies that were higher than the donors. However, this was only significant at $P=0.05$ for lines A and $\mathrm{C}$. If the 'exceptional' regenerant of line $\mathrm{F}$, mentioned above, were excluded from the analysis, the difference in means between donors and regenerants of line $\mathrm{F}$ was also significant at $P=0.05$.

Chiasma distribution. The average proportions of chiasmata (per $20 \mathrm{MI}$ p.m.c.s) that were distal, interstitial or proximal were also determined for all the donors and regenerated plants. As few cases of proximal chiasmata were observed they were pooled with interstitial chiasmata and the data for the proportion of chiasmata that were distal or non-distal analysed as described above (Table 3). In general, the inbred lines had higher proportions of distal chiasmata than the outbred lines which might be expected because of their lower chiasma frequency. There were significant differences in chiasma distribution between donors and regenerants in lines $\mathrm{B}, \mathrm{C}$ and $\mathrm{F}$ at $P=0.001$, in line $\mathrm{D}$ at $P=0.01$ and in $\mathrm{E}$ at $P=0.05$. In lines $\mathrm{B}, \mathrm{C}$ and $\mathrm{D}$, the regenerated plants had significantly higher frequencies of distal chiasmata than the donors, whereas in line $\mathrm{F}$ this trend was reversed.

Table 2 Comparisons of mean chiasma frequencies

\begin{tabular}{llllrl}
\hline \multicolumn{5}{c}{ Regenerant } \\
Line & Donor mean & mean & S.E.D. & d.f. & Probabilities \\
\hline A & 13.24 & 13.51 & 0.110 & 122 & 0.018 \\
B & 12.61 & 12.57 & 0.259 & 52 & 0.869 \\
C & 11.37 & 11.78 & 0.177 & 36 & 0.025 \\
D & 11.75 & 11.96 & 0.190 & 58 & 0.261 \\
E & 11.70 & 11.93 & 0.190 & 34 & 0.226 \\
F & 12.19 & 12.86 & 0.362 & 30 & 0.075 \\
\hline
\end{tabular}

S.E.D.: standard error of difference, d.f.: degrees of freedom.

Table 3 Comparisons of mean percentage distal chiasma

\begin{tabular}{lllrrr}
\hline \multicolumn{5}{c}{ Regenerant } \\
Line & Donor mean & mean & S.E.D. & d.f. & Probabilities \\
\hline A & 49.3 & 53.1 & 1.97 & 122 & 0.061 \\
B & 42.6 & 52.2 & 2.68 & 52 & $<0.001$ \\
C & 56.6 & 63.4 & 1.88 & 36 & $<0.001$ \\
D & 57.2 & 62.4 & 1.70 & 58 & 0.004 \\
E & 58.3 & 64.2 & 2.69 & 34 & 0.037 \\
F & 63.9 & 53.5 & 2.98 & 30 & 0.001 \\
\hline
\end{tabular}

S.E.D.: standard error of difference; d.f.: degrees of freedom. 


\section{Discussion}

Outbred and inbred lines of rye differed in shoot forming ability in regeneration from cultured immature embryos. This supports earlier reports indicating that morphogenetic response in rye is under genetic control (Linacero \& Vazquez, 1986; Bebeli et al., 1988). Difficulties were experienced in obtaining sufficient numbers of regenerants because the seed produced by the inbred lines were very small and in the outbred lines self-pollination drastically reduced the amount of seed set. The two outbred lines ( A and B) gave the best response but frequencies of shoot regeneration were low compared with previous reports (Bebeli et al., 1988; Zimny \& Lörz, 1989). In the outbred line A (cv. Ailés), the translocation heterozygotes did not differ in morphogenetic response compared with donors that were only bivalent-forming. In line $\mathrm{B}$, there were no clear differences in response of $0 \mathrm{~B}$ and $2 \mathrm{~B}$ containing plants in terms of the percentage of cultures that gave rise to shoots or the numbers of shoots produced.

Somaclonal variation in line A, as detected cytologically, was consistent with the characteristics of the cv. Ailés known from previous studies (Candela et al., 1979, 1982; Figueiras et al., 1983). Translocations were detected in two of 37 line A donors. In regeneration from donor $\mathrm{A} 7$, the translocation was unchanged by passage through a tissue culture phase. Similar results have been observed in wheat (Karp \& Maddock, 1984). In three of five outcrossed (bivalentforming) donors, some embryos gave regenerated plants which were translocation heterozygotes, indicating that the donors were polymorphic for the translocations. The high frequency of independent translocations found in regenerants from different embryos of a single donor (A22) is more likely, however, to be accounted for by de novo translocations occurring during culture rather than outcrossing. Further evidence for the occurrence of culture-induced translocations was obtained in regeneration from the selfed donor A25, where a translocation was observed in one of two plants regenerated from the same embryo. Also, in the outcrossed donor A7, where the translocation was expected to segregate among the different embryos but the constitution of regenerants from the same embryo should have been the same, one embryo gave one translocation heterozygote and three normal regenerated plants. It is more likely that this translocation was generated in the one plant rather than lost in the regeneration of the others. No evidence for somatic instability of the translocations was detected in the donor plants. Chromosome breakage in regenerants of cv. Ailés has been reported previously (Linacero \& Vazquez, 1992).

The translocation in one of the donors of line $\mathrm{B}$ must have occurred spontaneously because the plants were all selfed. This was confirmed from the pattern of secalin seed storage proteins revealed by sodium dodecyl sulphate-polyacrylamide gel electrophoresis (SDS-PAGE) (data not presented), which was entirely consistent with that obtained for the B line. In this study, there was no evidence that the $\mathrm{B}$ chromosomes were unstable in regeneration from tissue cultures, which is consistent with an earlier report in rye (Asami et al., 1976). Although a OB regenerant was obtained from a callus of a $2 \mathrm{~B}$ plant, it is not possible to determine whether the $\mathrm{B}$ chromosomes were lost during selfing or eliminated during callus growth, as shown in Cymbopogon martinii (Sreenath \& Jagadishchandra, 1988).

Two of the four inbred lines (C and $E$ ) were very stable during tissue culture in that no numerical or structural chromosome variation was observed in their regenerants. Line $\mathrm{D}$ was also stable, except for a single translocation heterozygote among the regenerants, which clearly arose during culture as it was not observed in the donor or in two other regenerants from the same cultured embryo. It is interesting that line F appeared to be the most unstable line in culture, because this line was described by Rees (1955) as showing chromosome breakage at meiosis. Two independently regenerated plants carried putative B chromosomes which were not observed in the donor plants or in other regenerants from the same cultures. The possibility that these were derived from outcrossing with line B was eliminated from the secalin patterns observed after SDS-PAGE (data not presented). The origin of the putative B chromosome types is not clear. A translocation was present in the donors of line $F$ and appeared in some regenerants. This serves to indicate the importance of screening the characteristics of the donor plants and of knowing the exact origins of each regenerant.

The results obtained here indicate that the nature of the cytological variation observed in regenerated plants differed among the six genotypes tested and was generally consistent with expectations based on the nature of the donors. However, somaclonal variation was still generated in inbred lines of rye which have been selfpollinated since 1926 , indicating that although some major determinants of instability may have been removed by inbreeding, the capacity of the lines for instability had not been completely eliminated during attainment of homozygosity and selection for selffertility.

An examination of chiasma frequency and distribution indicated that the regenerated plants generally had higher mean p.m.c. chiasma frequencies and more distally localized chiasmata, although only the latter differences were highly significant. Line $F$ was exceptional in that the localization trend was reversed. 
Genetic evidence indicating that recombination rates can be altered by passage through tissue culture has been described in potato and tomato (Sibi et al., 1984; Singsit et al., 1990; Compton \& Veilleux, 1991). It has been suggested that these changes could result from alterations in nuclear DNA content, structural chromosome changes or from the action of genetic modifiers but the data presented here suggest that they could also be attributed to a shift in chiasma distribution occurring as a result of tissue culture. Our results support the increasing evidence that tissue culture can cause changes in meiotic recombination. Even if the changes observed in the regenerated plants are not heritable (and preliminary studies of $23 \mathrm{R}_{2}$ plants suggest that they are), this aspect of somaclonal variation, which is less well documented than the occurrence of numerical and structural chromosome aberrations, has important implications for the levels of variation generated in the progenies of regenerated plants and therefore for studies of somaclonal variation in $\mathrm{R}_{2}$ generations.

\section{Acknowledgements}

The authors gratefully acknowledge the assistance of Ms G. M. Arnold at Long Ashton Research Station, IACR, for carrying out the statistical analyses. The authors also thank Miss S. H. Steele and Miss P. G. Owen at Rothamsted Experimental Station, U.K. and Long Ashton Research Station, IACR, U.K., respectively, for technical assistance. M. Puolimatka thanks Professor S. K. Pulli of the Agricultural Research Centre, Institute of Plant Breeding, Jokioinen, Finland and the Academy of Finland for financial support.

\section{References}

ASAMI, H., InOMATA, N. AND OKAMOTO, M. 1976. Chromosome variation in callus cells derived from Secale cereale L. with and without B chromosomes. Jap. J. Genet., 51, 297-303.

BEBELI, P. J., KARP, A. AND KALTSIKES, P. J. 1988. Plant regeneration and somaclonal variation from cultured immature embryos of sister lines of rye and triticale differing in their content of heterochromatin. Theor. Appl. Genet., 75, 929-936.

BEbELI, P. J., KARP, A. AND KAltsikes, P. J. 1990. Somaclonal variation from cultured immature embryos of sister lines of rye differing in heterochromatic content. Genome, 33, $177-183$.

BENZION, G. AND PHILLIPS, R. L. 1988. Cytogenetic stability of maize tissue cultures; a cell line pedigree analysis. Genome, 30, 318-325.

CANDElA, M., FIGUEIRAS, A. M. AND LACADENA, J. R. 1979. Maintenance of interchange heterozygosity in cultivated rye, Secale cereale L. Heredity, 42, 282-289.

CANDEla, M., Figueiras, A. M. AND LACADENA, J. R. 1982. Mutation-selection equilibrium as a possible cause of an interchange chromosome polymorphism in a cultivar of rye, Secale cereale L. Theor. Appl. Genet., 62, 321-324.
COMPTON, M. E. AND VEILleuX, R. E. 1991. Variation for genetic recombination among tomato plants regenerated from three tissue culture systems. Genome, 34, 810-817.

EIZENGA, G. C. 1989. Meiotic analysis of tall fescue somaclones. Genome, 32, 373-379.

FIGUEIRAS, A. M., CANDELA, M. AND LACADENA, J. R. 1983. Reciprocal translocations in Spanish and Portuguese natural populations of cultivated rye, Secale cereale L. Euphytica, 32, 493-498.

JOHNSON, S. S., PHLLIPS, R. L. AND RINES, H. W. 1987. Meiotic behaviour in progeny of tissue culture regenerated oat plants (Avena sativa L.) carrying near telocentric chromosomes. Genome, 29, 431-438.

KARP, A. 1992. On the current understanding of somaclonal variation. In: Miflin, B. J. (ed.), Oxford Surveys of Plant Molecular and Cell Biology, vol. 7, Oxford University Press, Oxford, pp. 1-58.

KARP, A. AND JONES, R. N. 1983. Cytogenetics of Lolium perenne. Part 2. Theor. Appl. Genet., 64, 137-145.

KARP, A. AND MADDOCK, S. E. 1984. Chromosome variation in wheat plants regenerated from cultured immature embryos. Theor. Appl. Genet., 67, 249-254.

KARP, A., STEELE, S. H., BREIMAN, A., SHEWRY, P. R., PARMAR, S. AND JONES, M. G. K. 1987. Minimal variation in barley plants regenerated from cultured immature embryos. Genome, 29, 405-412.

LARKIN, P. J. AND SCOWCROFT, W. R. 1981. Somaclonal variation - a novel source of variability from cell cultures from plant improvement. Theor. Appl. Genet., 60, 197-214.

LEE, M. AND PHILLIPS, R. L. 1988. The chromosomal basis of somaclonal variation. Ann. Rev. Plant Physiol. Plant Mol. Biol., 39, 413-437.

LINACERO, R. AND VAZQUEZ, A. M. 1986. Somatic embryogenesis and plant regeneration from leaf tissues of Secale cereale L. In: Horn, W., Jensen, C. J., Odenbach, W. and Schieder, O. (eds), Genetic Manipulation in Plant Breeding, Walter de Gruyter, Berlin, pp. 615-616.

LINACERO, R. AND VAZQUEZ, A. M. 1992. Cytogenetic variation in rye regenerated plants and their progeny. Genome, 35 , $428-430$.

REES, H. 1955. Genotypic control of chromosome behaviour in rye. I. Inbred lines. Heredity, 9, 93-116.

SIBI, M., BIGLARY, M. AND DEMARLY, Y. 1984. Increase in the rate of recombinants in tomato (Lycopersicon esculentum L.) after in vitro regeneration. Theor. Appl. Genet., 68, 317-321.

SINGSiT, C., VEILleUX, R. E. AND STERreT, S. B. 1990. Enhanced seed set and crossover frequency in regenerated potato plants following anther and callus culture. Genome, 33, $50-56$.

SREENATH, H. L. AND JAGADISHCHANDRA, K. s. 1988. In vivo and in vitro instability in palmarosa grass (Cymbopogon martinii var. motia). Genome, 30, 966-973.

SWEDLUND, B. AND VASIL, 1. K. 1985. Cytogenetic characterization of embryogenic callus and regenerated plants of Pennisetum americanum (L.) K. Schum. Theor. Appl. Genet., 69, 575-581.

ZIMNY, J. AND LÖRZ, H. 1989. High frequency of somatic embryogenesis and plant regeneration of rye (Secale cereale L.). Plant Breeding, 102, 89-100. 